\title{
STATE OF THE ART IN SEMICONDUCTOR DETECTORS.
}

\author{
P. Rehak \\ BNL- -43538 \\ Brookhaven Nat. Lab., Upton, N.Y., 11973. \\ DE90 005567
}

\section{E. Gatti}

Politecnico di Milano, 32 Piazza Leonardo da Vinci, 20133 Milano, Italy*. $\begin{array}{ccc}\text { JASTRACT JAN } 221990 & \end{array}$

The state of the art in semiconductor detectors for elementary particle physics and $\mathrm{X}$-ray astronomy is briefly reviewed. Semiconductor detectors are divided into two groups; i) classical semiconductor diode detectors and ii) semiconductor memory detectors. Principles of signal formation for both groups of detectors are described and their performance is compared. New developments of silicon detectors are reported here.

\section{INTRODUCTION}

There has always been a need for detectors providing precise position information in experimental particle physics. The momenta of charged particles are determined from the measurement of the curvature of tracks in a magnetic field. A better position resolution improves the measurement of the curvature and makes the determination of the particle momentum more precise. To improve the momentum measurement, however, it is also possible to increase the size of the apparatus and measure the curvature along longer segments of tracks.

This manuscript has been authored under contract number DE-AC02-76CH00016 with the U.S. Department of Energy. Accordingly, the U.S. Government retains a non-exclusive, royalty-free license to publish or reproduce the published form of this contribution, or allow others to do so, for U.S. Government purposes.

* This research is also supported by the Italian INFN and CNR.

Invited talk given at 4th Pisa Meeting on Advanced Detectors, Isola d'Elba La Biondola, Italy, May 21-26, 1989; to be published in Nucl。Instr. \& Meth. 


\section{DISCLAIMER}

This report was prepared as an account of work sponsored by an agency of the United States Government. Neither the United States Government nor any agency Thereof, nor any of their employees, makes any warranty, express or implied, or assumes any legal liability or responsibility for the accuracy, completeness, or usefulness of any information, apparatus, product, or process disclosed, or represents that its use would not infringe privately owned rights. Reference herein to any specific commercial product, process, or service by trade name, trademark, manufacturer, or otherwise does not necessarily constitute or imply its endorsement, recommendation, or favoring by the United States Government or any agency thereof. The views and opinions of authors expressed herein do not necessarily state or reflect those of the United States Government or any agency thereof. 


\section{DISCLAIMER}

Portions of this document may be illegible in electronic image products. Images are produced from the best available original document. 
The discovery of the new heavy lepton and of new quantum flavors about fifteen years ago-brought new requirements for the position resolution on tracking detectors. The life times of the $\tau$ lepton and of particles containing heavy flavors of charm or beauty are so short that they decay very close to the production point. In order to resolve the decay vertex from the production vertex, a position resolution better than several $\mu m$ is needed. When searching for secondary vertices indicating the decay of these particles there is no substitute for position resolution. Tracking detectors must be located as close as possible to the primary vertices and have the best position resolution in absolute terms.

Semiconductor detectors are well suited as high resolution position detectors. The density of the ionization by a minimum ionizing particle is by four orders of magnitude higher in semiconductors than in gases at atmospheric pressure. The high linear ionization density allows semiconductor detectors to be much thinner than the gas detectors. There is another advantage of semiconducting media for tracking as compared to gas media coming from the range of delta rays. The range of the delta rays in gas is much larger than in a semiconductor and the ionization may be spread away from the trajectory of the fast particle. The position information is degraded in the gas detector by the very process of the particle ionization. In semiconductors the ionization is confined within a $\mu m$ diameter column. This spread of the ionization is smaller than other fluctuations, which limit the position resolution of the present semiconductor detectors.

It is interesting to note that the use of semiconductor detectors for position measurements is relatively recent. The traditional use of semiconductor detectors was in the field of nuclear spectroscopy. The conversion of the released energy into signal charge is more efficient in the semiconductors than in gases. The same released energy produces about ten times more charges in semiconductors than in a gas. The higher number of fret cliarges together with a lower value of the Fano factor in semiconductors leads to a smaller relative fluctuation and a better intrinsic energy resolution. Presently semiconductor detectors are used almost exclusively for the low energy $X$-ray spectroscopy and as detectors in a new field of low energy $X$-ray astronomy.

This contribution is organized as follows: in the second section we will divide the present semiconductor detectors into two groups (classical and memory semiconductor detectors) according the mechanism of the charge removal from the detector. In the third 
section we will describe classical semiconductor detectors and describe the most important developments in recent years. In the fourth section we will introduce a newer kind of semiconductor detectors called memory semiconductor detectors. We will stress the principles of operation and report the progress during the recent years.

The fifth Section will attempt to compare the performance of classical and memory semiconductor detectors. We will see that the performance of the classical semiconductor detectors depends mainly on the performance of the read-out electronics while the performance of the memory detectors depends mainly on the parameters of the detector itself.

\section{CLASSIFICATION OF SEMICONDÜCTOR DETECTORS}

Semiconductor detectors can be divided into several groups according to different criteria. In this contribution we will divide semiconductor detectors used up to 1989 into two large groups distinguished by the method of the charge removal from their bulk. Semiconductor detectors can be thus divided into the two following groups:

1. classical semiconductor detectors and

2. semiconductor memory detectors.

\section{CLASSICAL SEMICONDUCTOR DETECTORS}

The first semiconductor detectors developed in $1951^{1}$, were classical diude detectors. Today these detectors are widely used as

1. X-ray detectors

3. Photudiudes

3. Microstrip Detectors

4. Pad Detectors

5. One kind of Pixel Detectors etc.

The cross section of a classical semiconductor detector is shown in Fig. 3.1. As an example, $n$-type silicon in form of a thin disk (wafer) is shown. The silicon has a rectifying $p^{+} n$ junction on the upper face and an $n n^{+}$junction acting as a non-injecting ohmic contact on the lower face. The rectifying junction is reverse biased at a sufficiently high 


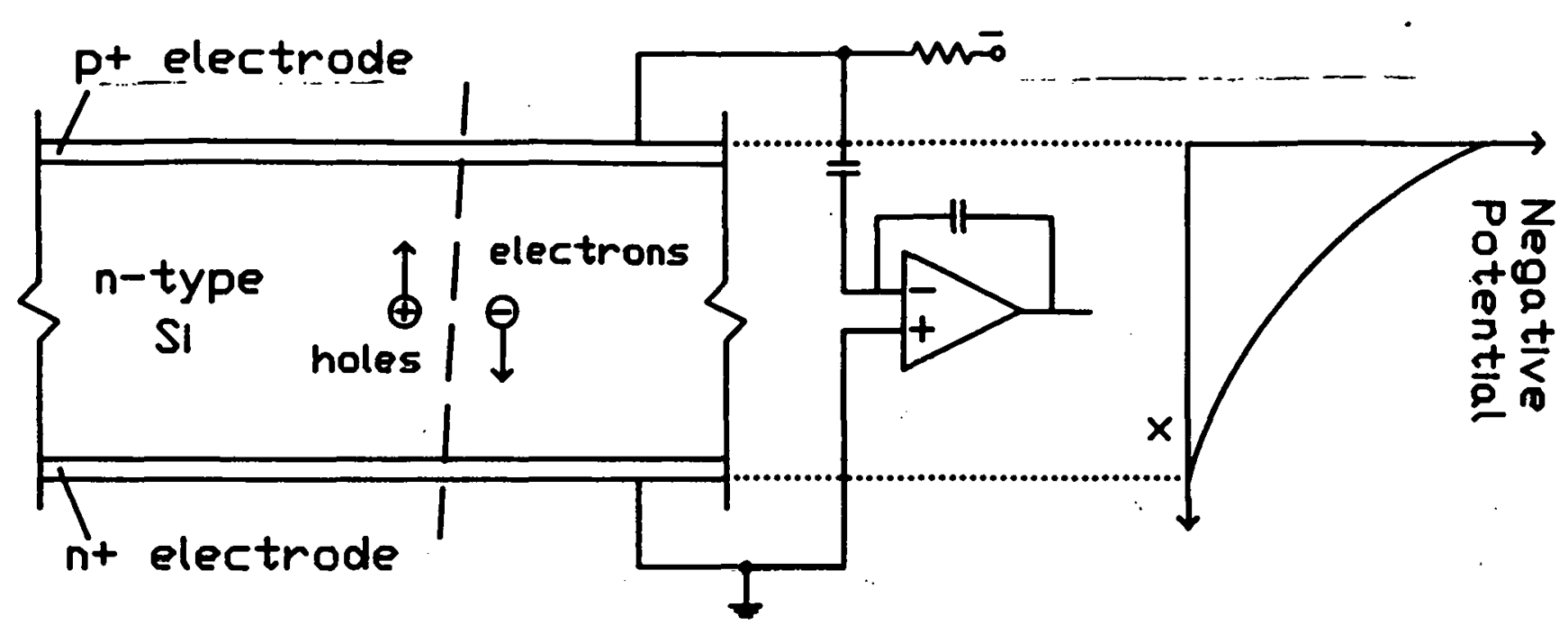

Figure 3.1: a) Cross section of a classical semiconductor detector. b) Negative potential in the bulk of a classical detector.

voltage to remove all free charges between the two surface electrodes (complete depletion of the silicon bulk). In the depleted bulk a space charge is present due to the ionized donors fixed in the silicon lattice. The electric field in the bulk is created by this space charge and the surface layer charges on the junctions.

The presence of the field does not cause a strong current flow through the detector. Only a small reverse current of both junctions and a small current of carriers thermally generated in the bulk flows through the detector. The depletion and the electric field are essential for the functioning of the semiconductor detector. Electron-hole pairs generated by the radiation move apart and towards the two junctions in the electric field. The motion of the charges within the bulk of the detector induces a signal current in the external circuit which includes a charge sensitive preamplifier. The total charge measured by the preamplifier is equal to the charge produced by the ionizing particles in the detector when all electrons and holes reach the $n n^{+}$and $p^{+} n$ junctions respectively.

In the parallel plate geometry of Fig. 3.1 the field is normal to the plates. The negative potential or the potential energy of electrons in the bulk is also shown in Fig. 3.1. The motion of carriers in silicon can be easily visualized with the help of pictures of the negative potential. Electrons move down as small balls without inertia, while holes moves up as 
bubbles. The charges of both polarities spend a minimal time within the detector bulk. The fast removal of all charges produced by ientzation from the-bulk of the detector is themain feature of classical semiconductor detectors.

In spite of a very short carrier collection time in classical semiconductor detectors the total read-out time is usually long. The same electrode geometry of classical semiconductor detectors which provides the fastest removal of charge from the bulk of the detector leads to a large anode capacitance of the detector. A large anode capacitance makes the noise of the preamplifier important. There is no signal amplification in semiconductor detectors and in order to obtain a sufficient signal to noise ratio, the bandwidth of the processing electronics must be limited. The rise time of the overall response is therefore much longer than the collcetion time of the carriers in the detector. The duration of the proressing time rather than the carrier collection time limits the rate capability of classical semiconductor detectors.

The position resolution in this kind of detectors is obtained simply by the division of at least one junction into strips. Each strip is connected to its owa preamplifier. A very large number of preamplifiers is needed to read the position information from strip detectors.

The most important progress in strip detectors (apart from the development of the VLSI read-out which is the subject of another session) are the recently introduced double sided strip detectors ${ }^{2}$. These detectors have both junctions divided into parallel strips; strips on the upper junction are oriented perpendicularly to the strips on the lower junction. Each strip on both sides is connected to its own preamplifier. (Charge division methode which decreases the number of preamplifier by a factor of few is usuully used.) Doublc sided strip detectors are advantageous in detector systems where minimization of scattering material is important. Moreover, electrons and holes are generated in pairs by ionizing particles and the charge detected in an upper strip should be exactly the suwe us the charge detected in a lower strip. 'The preamplifier noise distort the perfect equalily of thcsc charges. Nevertheless, the use of the signal sharges decreases the matching problems of multiparticle detection in a single strip detector.

The largest problem in the realization of working double sided strip detectors is the presence of positive charges at the $\mathrm{Si}-\mathrm{SiO}_{2}$ interface. These charges arc compensated by an accumulation layer of electrons in silicon right under the interface. Electrons of 
the accumulation layer are free to move and a detector with a simple division of the $n n^{+}$ junction into individual strips would have all strips shorted together. Detectors descibed in Ref. 2 have an additional p-implantation between adjacent $n^{+}$strips to interrupt the accumulation layer and electrically separate the strips. There are also other methods to achieve the electrical separation of strips $^{3}$.

\section{SEMICONDUCTOR MEMORY DETECTORS}

In semiconductor memory detectors charges produced by ionizing particle are not removed and detected immediately from the detector as it was the case in classical detectors. In a memory detector at least one kind of charge carriers (usually electrons) is stored within the volume of the detector. The stored carriers are transported in a controlled way in a direction parallel to the wafer surface to a read-out electrode. The position information is retained during the carrier transport. The storage of the information can be often regarded as a first part of the signal pipeline. Moreover, the capacitance of the read-out electrode can be very small leading to a low noise contribution of the preamplifier.

The examples of semiconductor memory detectors are:

1. Surface and Buried Channel Charge Coupled Devices (CCDs)

2. Fully Depleted CCDs

3. Semiconductor Drift Detectors

4. Small Capacitance X-ray Detectors and Photodiodes

5. Some Pixel Detectors, etc.

CCDs were proposed in $1970^{4}$ as signal processing devices with a second use as optical imagers. There is only a short step between optical imagers and detectors of charged particles $^{5}$. The following subsections will describe principles of operation of fully depleted CCDs and semiconductor drift detectors.

\subsection{Fully Depleted Charge Coupled Devices}

The electrode structure of any of the semiconductor memory detectors is more complicated than the structure of classical detectors.

Fig. 4.1 shows the cross section of a fully depleted Charge Coupled Device (CCD), a very good example of a memory semiconductor detector. There are rectifying junctions on 


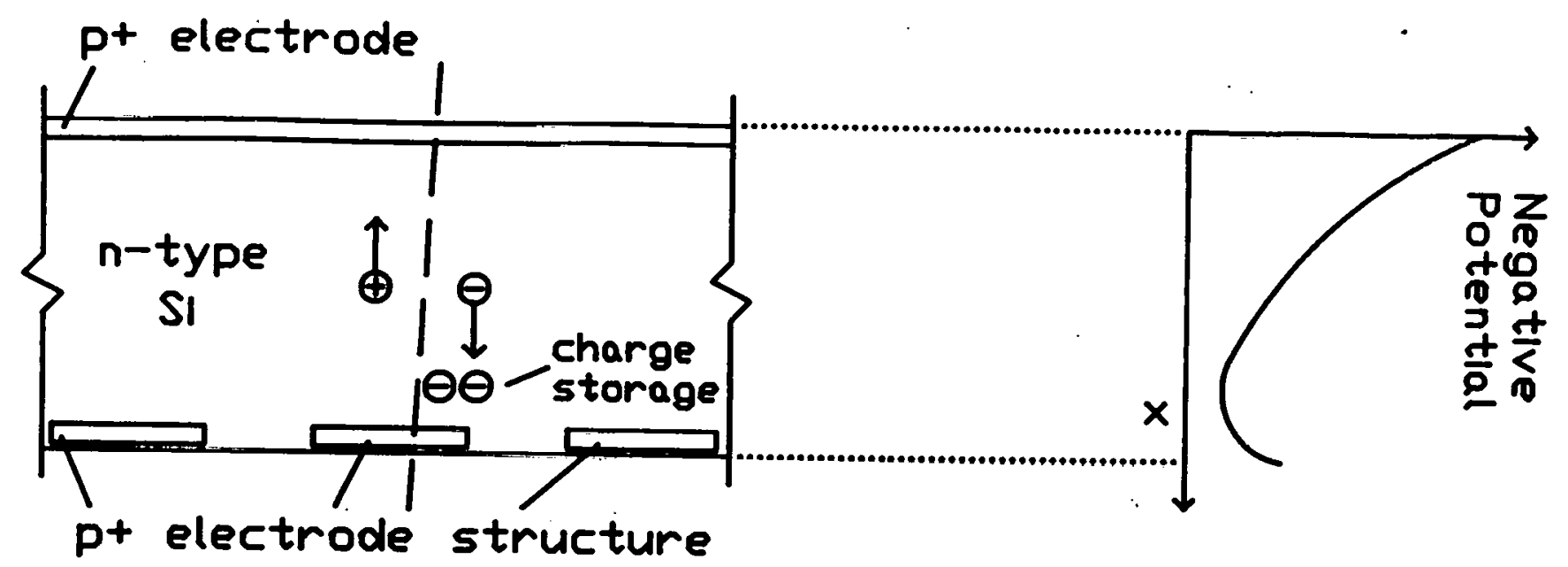

Figure 4.1: Cross section of a Fully Depleted Charge Coupled Device illustrating general features of memory detectors.

both upper and lnwer faces of the wafer. The wafer is supposed again to be of an $n$-type semiconductor and is completely depleted of mobile electrons. The space charge of the ionized donors in the silicon lattice together with the voltages applied to the upper and to the lower junctions produce an electric field. The negative potential of the electric field in a cross-section through the bulk of a fully depleted CCD is shown on the right hand side of Fig. 4.1.

Letu us asoumo that a minimum innizing particle crossed lle COD and produced a culumi of electron hole pairs. Holes mowe tnwnrifi one of the rectifying, junctions, but electrons move to the point of minimum negative potential where they are stored. The potential in a real fully depleted CCD is engineered in such a way that electrons are confined in this region and cannot diffuse in any direction. The picture of electron confinement in two dimensions is showin in Fig. 4.2. A fully depleted CCD contains many (of the order of $10^{6}$ ) such regions or elementary memory units (pixels) arranged in a two-dimensional matrix. Hilectrons produced by the meulivied minimim ionizing particle are stored in one or in a few pixels while the other pixels are generally empty.

There is no external circuit and no preamplifier shown in Fig. 4.1. The signal produced by the motion of electrons falling in one pixel and holes moving lo the surface junction 


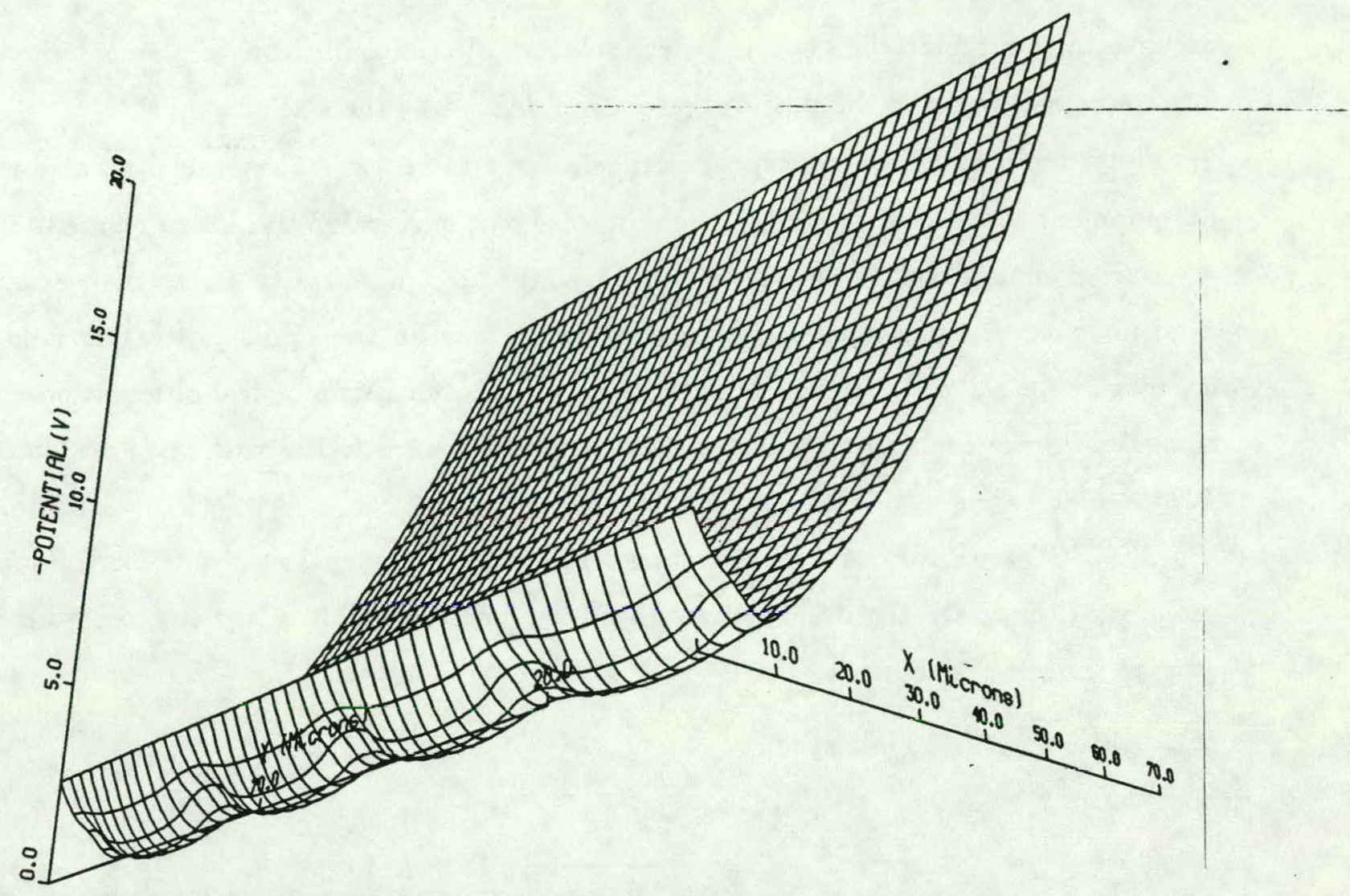

Figure 4.2: Negative potential in a Fully Depleted CCD in two dimensions. Local minima confine the signal electrons and define individual pixels.

is not read-out. There is an electrode structure, called a peristaltic pump, implemented on the lower face of a fully depleted CCD. When a sequence of appropriate voltages is applied to the shown electrode structure, the charges stored in the bulk are shifted from one pixel to the next one without any loss of electrons. Finally electrons are pumped into the last memory cell where their presence affects a read-out preamplifier. The pumping of electrons from the bulk to the reading electrode also cleans all pixels from any background electrons produced by thermal generation in the bulk or at the junctions.

There is only one preamplifier for the whole CCD. The position of the passing particle is measured when the corresponding pixel is read. The capacitance of the read-out cell of the CCD is very small so the preamplifier noise is kept at the minimum, hence CCDs have a very good signal to noise ratio. These devices are ideally suited for X-ray astronomy 
because they combine the good energy resolution of semiconductors implemented with a low noise-read-out-and the position resolution defined by the size of a pixel.

Fully depleted CCDs were proposed relatively recently ${ }^{\circ}$. They are presently under development to be used as focal plane detectors on the X-ray Multi-Mirror Satellite ${ }^{7}$.

Buried channel CCDs have reached a very high level of maturity due to their extensive use in consumer electronics as imagers in camcorders, in the signal processing field and as imagers for scientific applications. The best CCDs for astronomical observations reach equivalent noise charge down below a 1 electron level and CCDs with the granularity of $4096 \times 4096$ pixels were produced ${ }^{8}$.

The CCDs are the memory detectors which store the signal electrons for the longest amount of time. On the other extreme we have semiconductor drift detectors where the storage time is the shortest.

\subsection{Silicon Drift Detectors}

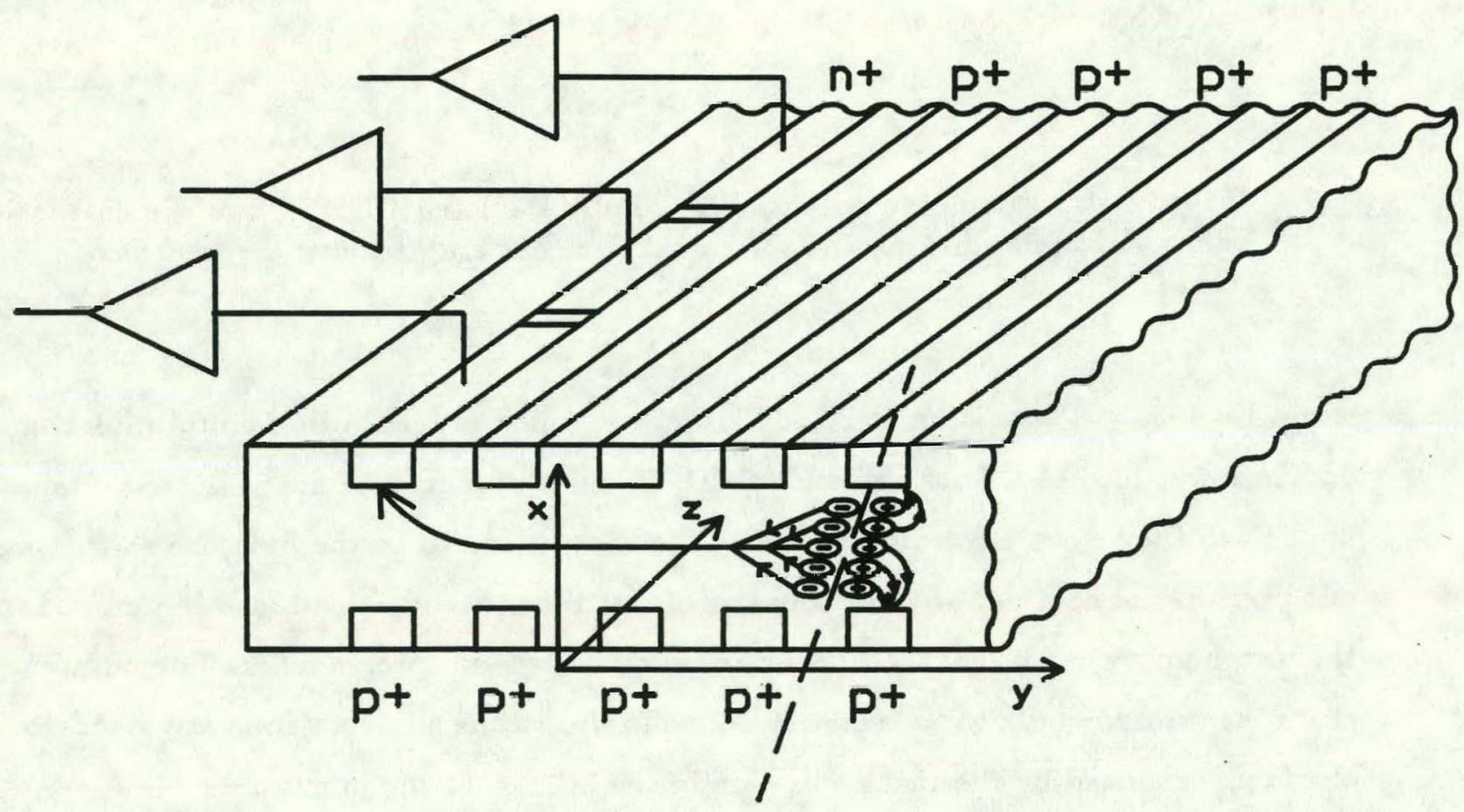

Figure 4.3: Perspective view (not to scale) of a semiconductor drift detector. Electrons created by an ionizing particle are transported long distances parallel to the detector surface. The anode is divided into short segments to measure the second coordinate. 
A perspective view of the drift detector ${ }^{\circ}$ is shown in Fig. 4.3. The volume of the detector is fully depleted of mobile electrons. The field created by the remaining fixed charges confines electrons generated by an ionizing particle in a buried potential channel. An electrostatic field parallel to the surface is superimposed. This field transports electrons created by a particle passage along the buried channel towards a collecting anode. The transit time of the electrons inside the detector measures the distance of an incident particle from the anode.

The exact shape of the electric potential in drift detectors requires some explanation. The cross-section of the negative potential shown in Fig. 4.1 is the solution of Poisson's equation in one dimension

$$
\frac{d^{2} \phi(x)}{d x^{2}}=\frac{\rho(x)}{\epsilon}
$$

where $\phi(x)$ is the negative potential, $x$ is the coordinate perpendicular to the surface plates of the detector, $\rho$ is the space charge density and $\epsilon$ is the absolute dielectric constant of silicon. The potentials applied at the two faces determine the two integration constants. The negative potential shown in Fig. 3.1 and in Fig. 4.1 are parabolas corresponding to the solution of Eq. (4.1) in a simple case where $\rho$ is independent of $x$.

While the electric field of a classical diode detector can be understood in a one dimensional model, the electric field of drift (memory) detectors must be described at least in two dimensions as it was already obvious from Fig. 4.2. A solution of Poisson's equation in two dimensions:

$$
\frac{d^{2} \phi(x, y)}{d x^{2}}+\frac{d^{2} \phi(x, y)}{d y^{2}}=\frac{\rho(x, y)}{\epsilon}
$$

is shown in Fig. 4.4. There the negative potential in the drift region of the detector is displayed. Poisson's equation in two dimensions Eq. (4.2) is satisfied by adding to the parabolic solution in $x$ of Eq. (4.1) a linear term $U_{y}=E_{d} \cdot y$, where $E_{d}$ is the drift field in the drift direction $y$. To realize the potential shown on Fig. 4.4 we have to impose the same linear $y$ dependance of the potential on both surfaces of the detector.

Fig. 4.4 shows a relatively strong electric field at the surfaces of the detector. Any electrons present on the surface would be injected into the valley. To prevent the electron injection, electrodes at both surfaces must be $p^{+}$type ( $p^{+} n$ rectifying junctions). This is a common requirement for all fully depleted memory detectors. 


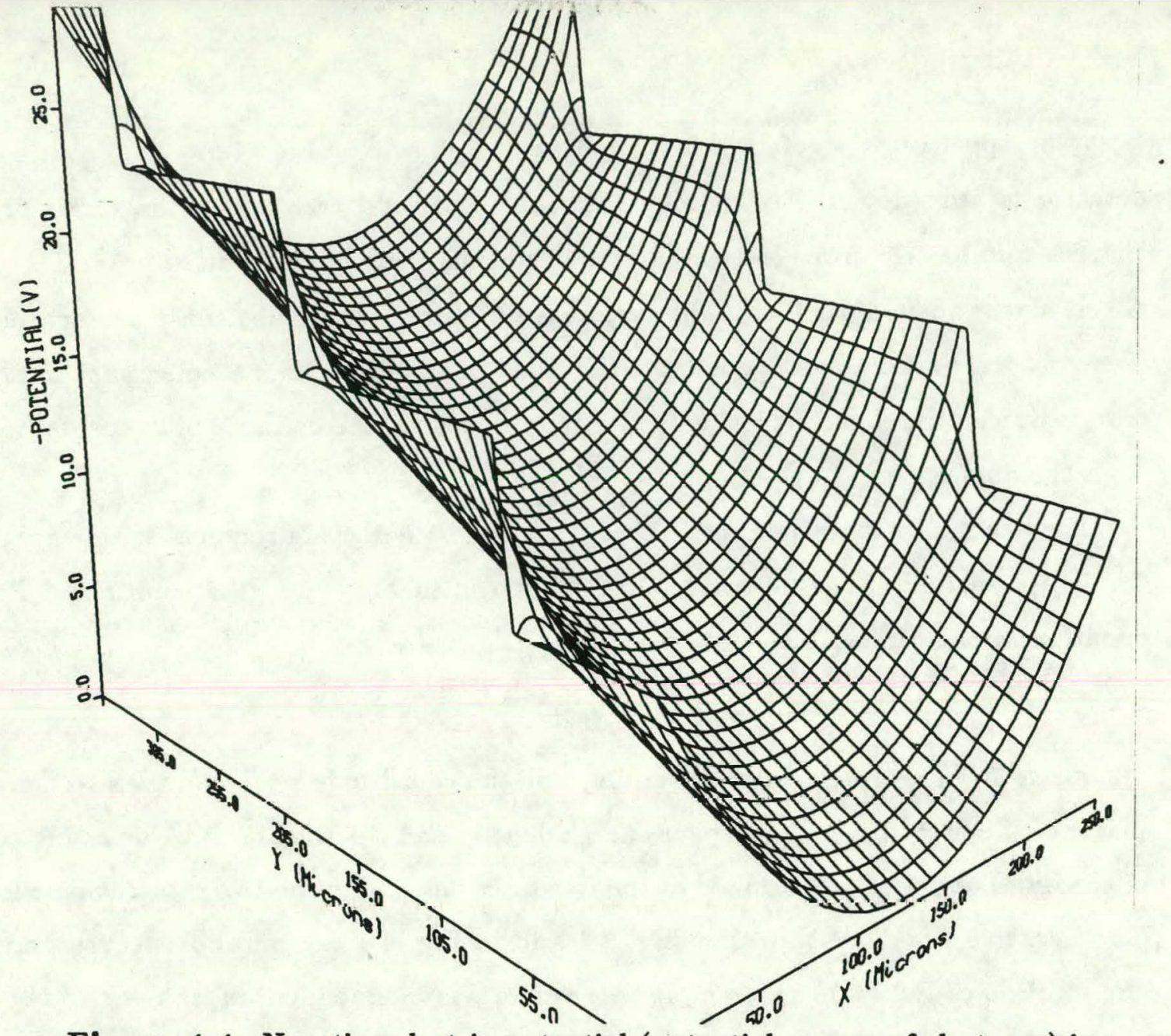

Figure 4.4: Negative electric potential (potential energy of electrons) in a linear drift detector. Surfaces of the detector are in planes $x=0$ and $x=300 \mu m$. Reverse biased $p^{+}$electrodes have the potential imposed by a voltage divider.

To realize the linear term in the potential of a drift detector, the $p^{+}$electrodes are segmented into strips. The inclined parabolic shape of the potential energy for electrons (negative potential) collects electrons in the middle of the bulk and transports them to the anode.

The anode region of the detector is shown in Fig. 4.5. Potentials are applied at the surfaces in a way to bend the bottom of the valley towards the surface collecting anode. The potential energy of the anode as shown is at the lowest potential energy in the active region of the drift detector. All electrons generated in the bulk arrive at the anode. Thus the detector is maintained in full depletion during the operation. 


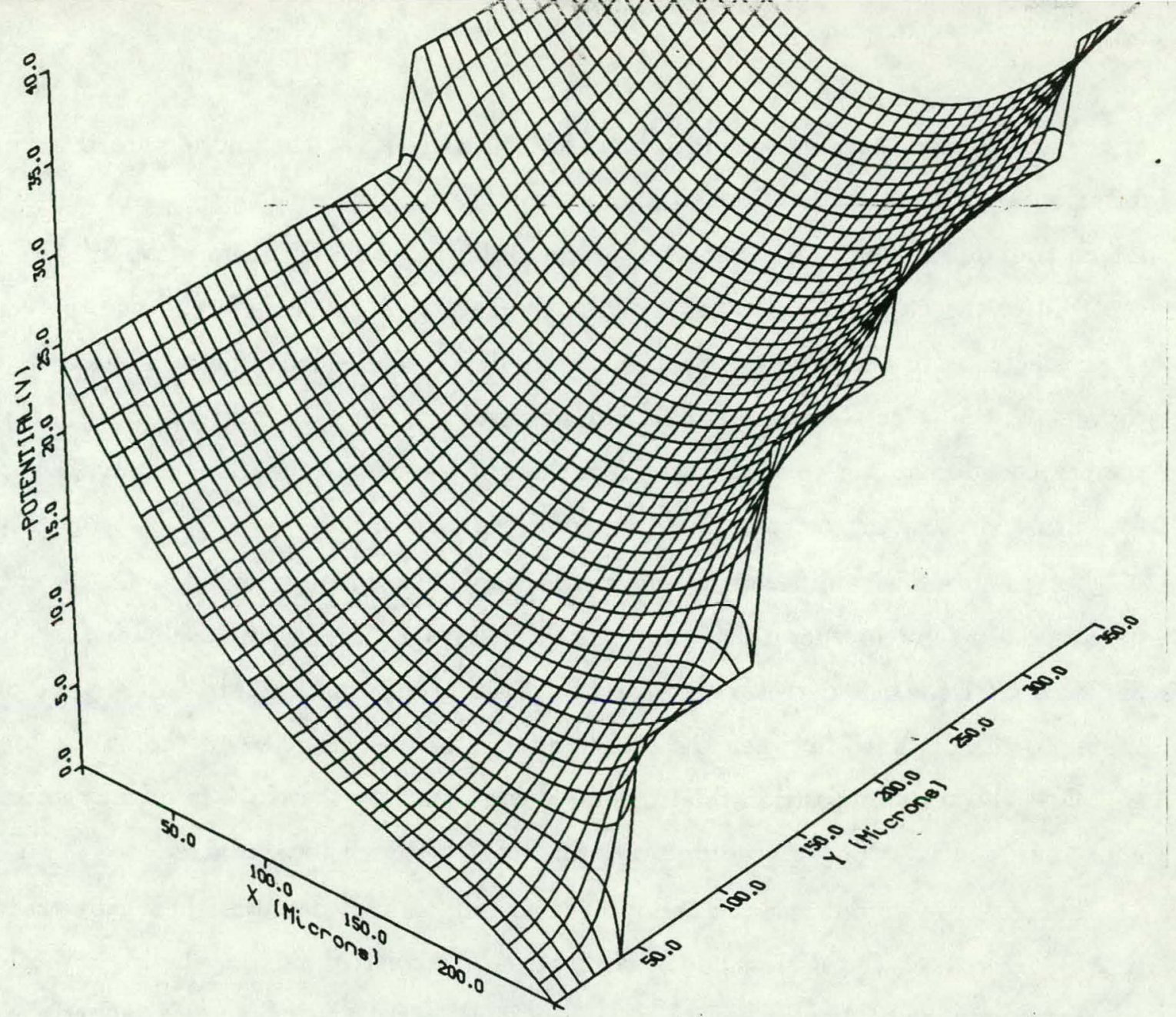

Figure 4.5: Negative electric potential in the anode region of a drift detector.

The electrodes, apart from imposing the desired surface potential on the drift detector, act as an electrostatic shield for drifting electrons. Electrons induce the signal charge only when they arrive to a close proximity of the anode. The drift time is the difference between the arrival time sensed by the anode and the time passage of the particle detected by other detectors of the experiment or provided by the accelerator. The information about the coordinate perpendicular to the drift direction is not lost during the transport in a linear drift detector. Fig. 4.3 shows that the anode divided into short segments provides also the position information in the direction perpendicular to the drift. The surface of the individual anodes is small and independent of the size of the detector. Thus the anode capacitance is very small as it is the case for all memory detectors.

The amplifier noise is not the only limitation for the position resolution of drift detectors $^{9}$. The detector leakage current and the statistical fluctuations of the pulse shape 
at the anode also limit the resolution. Electrons arriving at the anode spread because of diffusion and the electrostatic repulsion during the drift time. The sigma of the diffusion for an individual electron is relatively large $(150 \mu \mathrm{m}$ for a drift time of about $1 \mu s)$. The centroid of the electron pulse has a sigma reduced by $\sqrt{20000}$. We can use the centroid of all electrons to measure the position of particles. The electric field in the drift detector is such that electrons created contemporary at a given $y$-coordinate, independently of their $x$-coordinate, arrive at the anode in the same average time. The achievable position resolution is a few $\mu m$ for a drift distance of several $m m .^{10}$

Let us summarize differences and similarities between fully depleted CCDs and drift detectors. In semiconductor drift detectors the storage time is much shorter than in fully depleled CCDs. Drift detcetors convert the position information inten a. drift time proportional to the distance between the crossing point of the particle and the anode location. In CCDs the time to position relationship is controlled by the clock frequency and by the chosen sequential order of reading pixels in the two-dimensional matrix.

There were two important developments in silicon drift detectors in recent years. The first progress was the design and a realization of a drift detector where all the leakage current produced at the $\mathrm{Si}-\mathrm{SiO}_{2}$ interface is collected at sink anodes rather than contributing to the detector leakage current ${ }^{11}$. The parallel noise of the detector is decreased by a factor of five for the present detectors. Moreover, detectors are less sensitive to the surface damage and may be radiation harder.

'The second progress in the fleld of drift delecturs is the integration of the preamplifier directly on the silicon of the detector.

The realization of the first amplification stage directly on the wafer of the detector has two advantages

1. the input transistor can be made small enough to match the small detector capacitance.

2. stray capacitances due to the connection between the detector anode and the first transistor can be kept at a minimum.

These advantages are the most important for silicon drift detectors where capacitances of individual anodes are below $0.1 p F$. Any connection to an external preamplifier results in 
a highly mismatched system. The realization of matched preamplifiers with a minimum stray capacitzoce decreasses the noise of the detector-preamplifier system, resuilting in a substantial improvement of energy and position resolution of all silicon memory detectors. The first prototype of the integrated electronics is working ${ }^{12}$ and in the very near future there will be memory detectors with better performance than reported up to now ${ }^{13}$.

\section{COMPARISON OF CLASSICAL AND MEMORY SEMICONDUCTOR}

\section{DETECTORS}

At a first look one can have the following impression when trying to compare classical and memory semiconductor detectors: Memory detectors can achieve a better performance due to their much lower anode capacitance, however, the classical detectors are faster. In reality, in almost all practical situations, measurement systems based on memory detectors are not only more precise but also faster than systems based on classical detectors. We will illustrate our point for both energy and position measurements with semiconductor detectors.

When the spectrum of energy of radiation is measured, the speed of the system is expressed by the rate of pulses the system can process without pileups. The pulse duration limits the processing rate. The duration of the pulse in classical detectors is not the carrier collection time, but the time required to decrease the amplifier noise down to an acceptable level. The processing time for systems based on classical semiconductor detectors is typically a few tens of $\mu s$ with the corresponding maximal rate of a few tens of $k H z$.

For silicon drift detectors a longer drift (store) time does not limit the rate. Electrons drifting toward the anode have their charges screened by the electrode structure and do not interfere with the signal processing at the anode. The important time is the diffusion time, that is, the time it takes to collect the full charge at the anode. It can be shown that the diffusion time $t_{\text {diff }}$ is given by:

$$
t_{\text {diff }}=8 \cdot t_{\text {drift }} \sqrt{\frac{2 \cdot k T}{U_{\text {drift }} \cdot q}}
$$

where $t_{d r i f t}$ is the drift time, $U_{d r i f t}$ is the absolute value of the drift voltage and $k T / q$ is the thermal voltage equal to $0.025 \mathrm{~V}$. For practical low capacitance $\mathrm{X}$-ray drift detectors the 
diffusion time is about $100 \mathrm{~ns}$. The shaping time is again about $100 \mathrm{~ns}$ due to the extremely low capacitance of the detector anode. We see that the rate capability of low capacitance drift detectors is between a factor of 10 and 100 greater than the rate capability of classical detectors.

The comparison of position sensing for classical and memory detectors gives similar results. We can compare the performance of a microstrip detector with that of a single anode linear drift detector. To obtain similar position resolutions a classical microstrip detector needs hundreds of read-out preamplifiers rather than the single preamplifier of a drift detector. A practical microstrip read-out system (microplexer or equivalent) has, after a stage of amplification, a parallel to serial conversion to keep the number of readuuls at a reasonable level. The equivalent of the parallel to serial conversion is intrinsically present in silicon drift detectors. As it turned out the read-out speed of a multiplexer is wuch slower than the drift time of electrons in the linear drift detector.

We would like to stress again that the maximum drift time is not the dead time of the silicon drift detector. The signals are stored within the detector for the duration of their drift. In a high rate environment (Superconducting Super Collider - SSC, for example) a silicon drift detector stores at any given time hits of particles originating from different events.

Fig. 5.1 shows a simple way how to resolve particles originating from different events. Two silicon drift detectors are placed parallel one to another at a small distance.The drift fields in the two deteotors are arranged in such a way lhat electrons drift in opposite directions in these two detectors. Following some easy calculations of Fig. 5.1 we see that there is a position independent relation between the mean value of the drift times measured in both detectors and the time of the particle crossing. The time resolution of each drift detector is better than $1 n s$. The mean value of the drift times of the pair of drift detectors and hence the event timing can be determined with a resolution better than $1 n s$. There are not many position sensing detectors with this kind of time resolution.

We should now stress that there is no penalty to pay by using two silicon drift detectors instead of one. Once the association of hits with an event is completed we have two independent measures of points leading to a vector. This "vector" geometry is most likely preferable also from the pattern recognition point of view. 


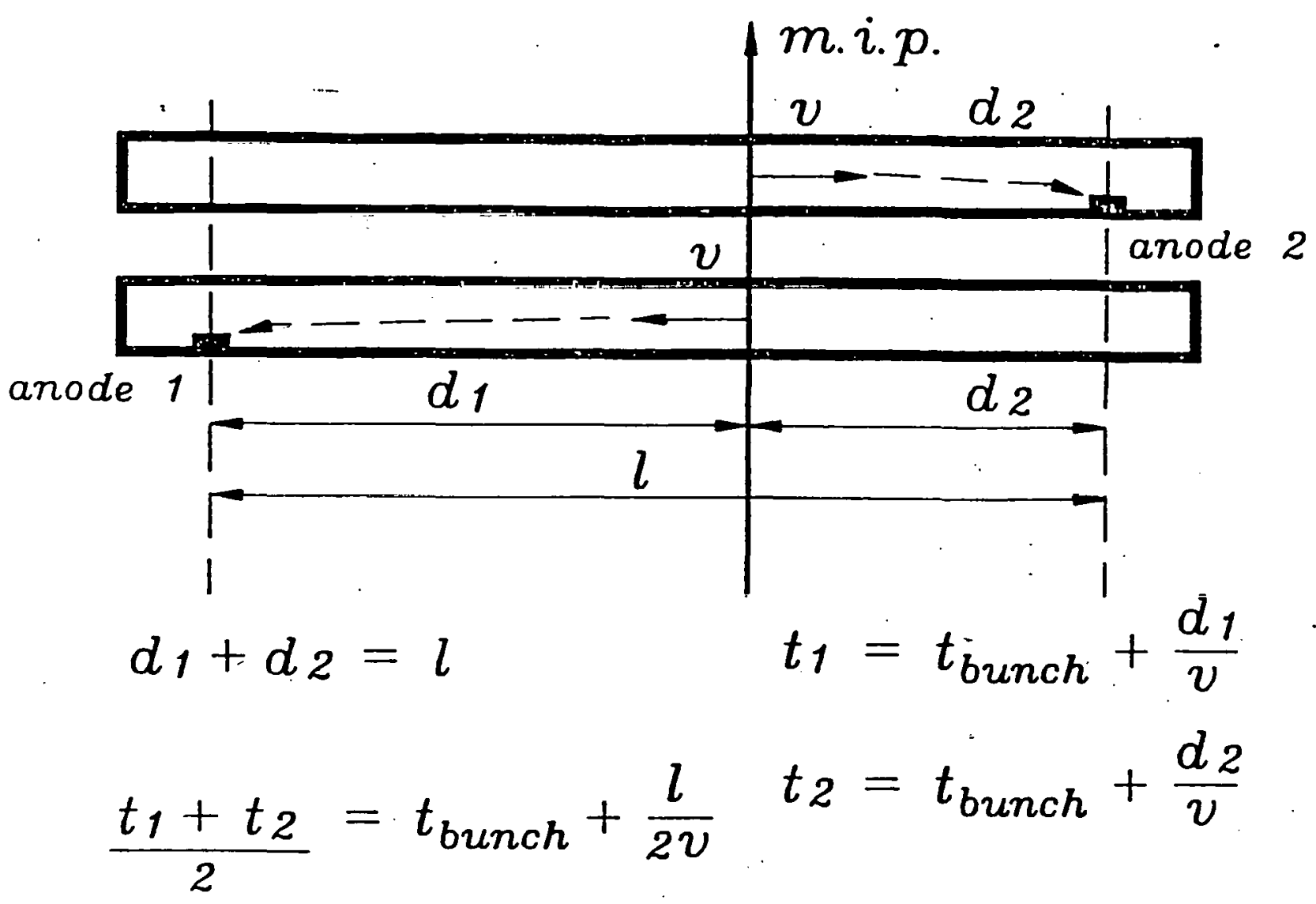

Figure 5.1: View (not to scale) of two silicon drift detectors placed in such a way that a particle has to cross both detectors and the produced electrons are drifting in opposite directions with the same speed $v$. The sum of drift distances for any position of the incident particle is constant and equals to the distance between anode1 and anode2. The average of the two arrival times gives the time of the event.

Multianode drift detectors and CCDs provide unambiguous two coordinate measurement with the position resolution in a few $\mu m$ region. There are no other detectors to which we could compare them.

Let us compare the performance of drift and classical semiconductor detectors used as sampling detectors in a shower counter. Fig. 5.2 in its upper part shows several particles traversing the detectors where only the total charge released is to be measured. Anode of the drift detector sees the charge arriving in separated clouds during the drift time of the device. The classical detector sees all charges at once (middle part of Fig. 5.2). 

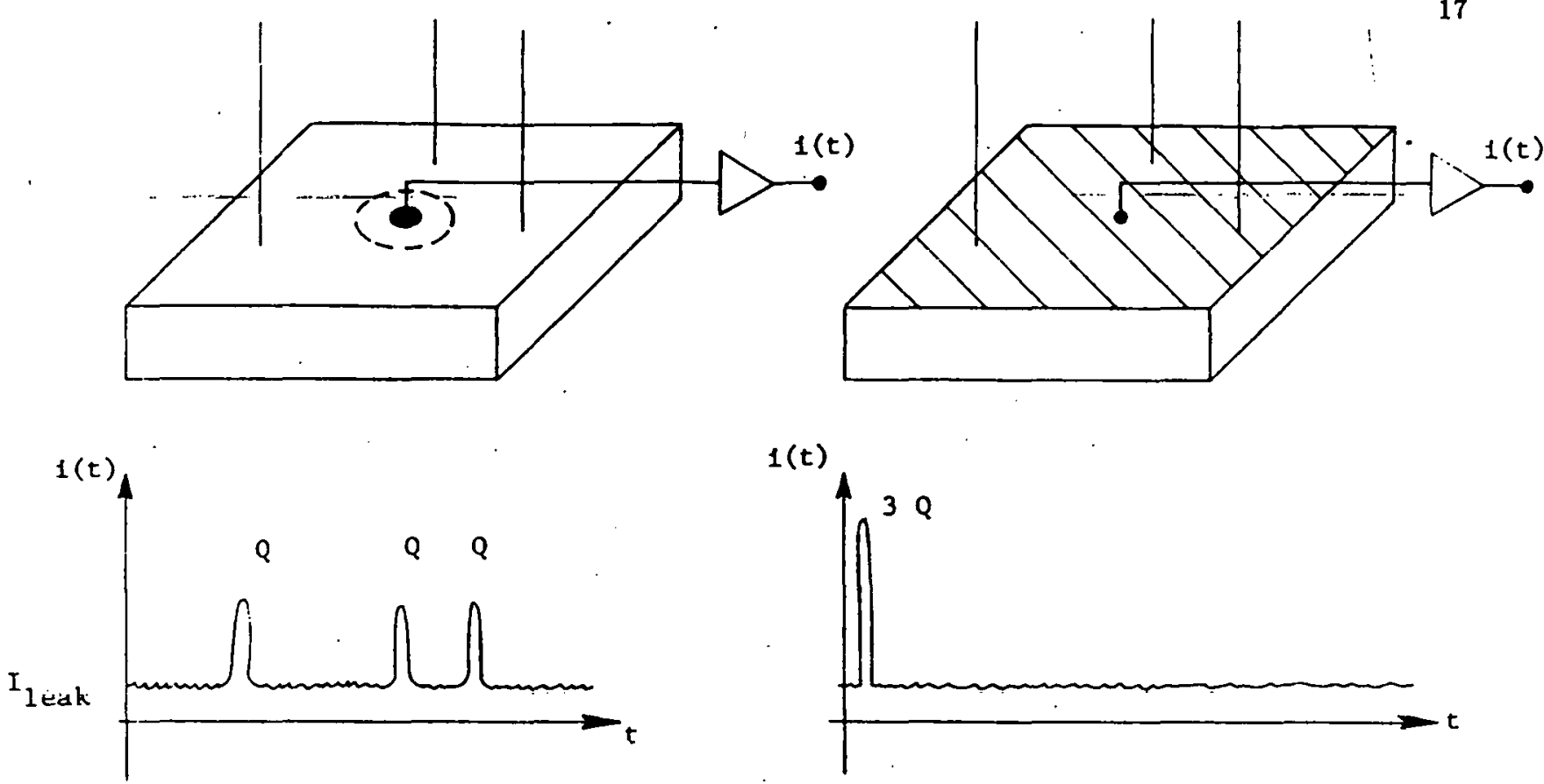

$1(t)$
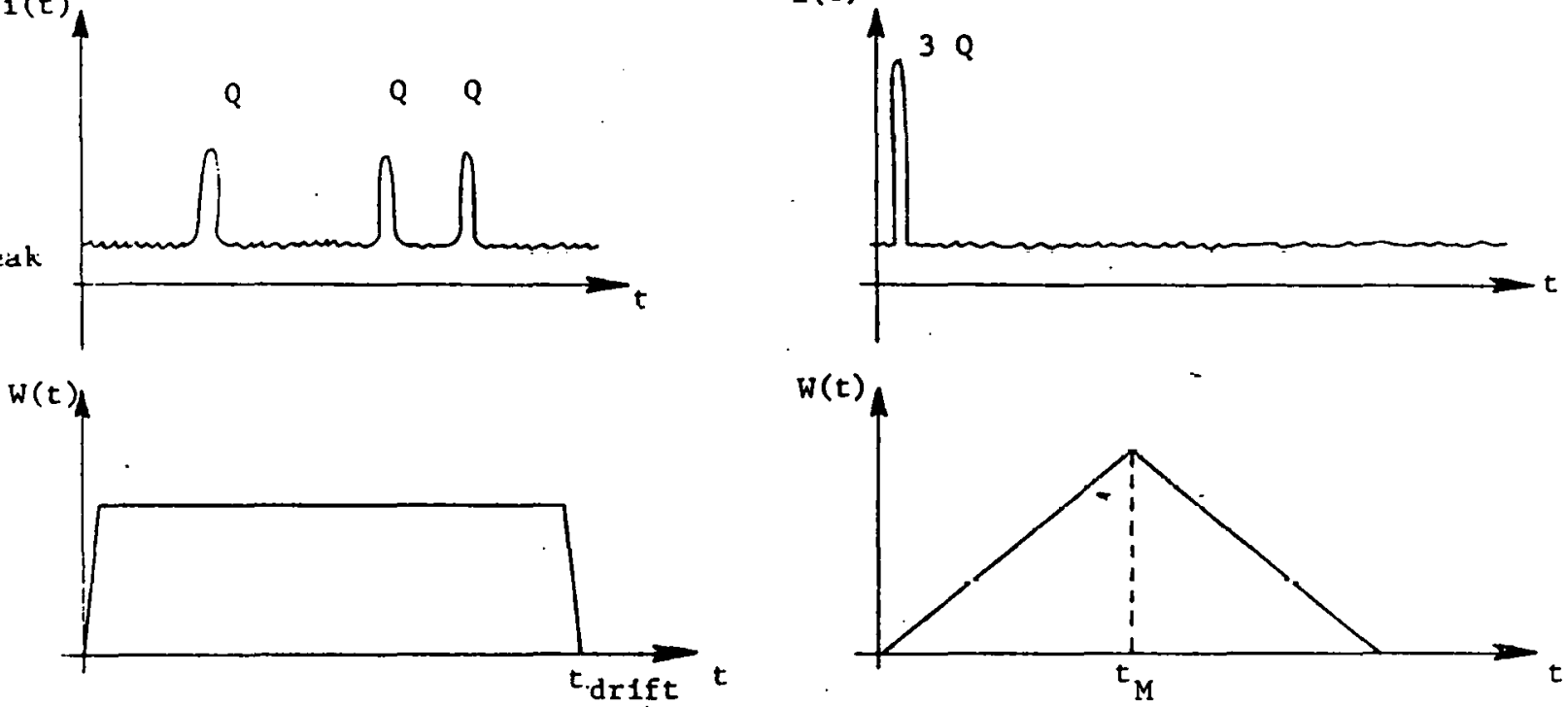

Figure 5.2: Signal processing of a drift (left hand side) and a classical (r.h.s.) semiconductor detectors as sampling detectors in a shower counter. Three minimum ionizing particle incident on detectors (upper part). Current signal waveform at the corresponding anodes (middle part). Weighting functions for an optimum signal processing (lower part).

The weighting function of the drift detector must have the flat part at least equal to the maximum drift time to measure the charge independently from the point of the origin. The weighting function of the classical detector does not need a flat top (lower part of Fig. 5.2).

The series noise of the drift detector is negligible. The equivalent noise charge (ENC) is due to the leakage current of the detector.

$$
(E N C)^{2}=I_{l e a k} \cdot q \cdot t_{d r i f t}
$$

where $I_{\text {leak }}$ is the leakage current, $q$ is the electronic charge and $t_{d r i f t}$ is the maximum drift time. 
The parallel noise in the classical detector is less important as compared to the parallel noise of the drift detector due to the different weighting funetion and can be neglected relative to the series noise of the preamplifier. After a straightforward calculation and assuming an ideal matching for the classical detector we can write

$$
(E N C)^{2}=\frac{16 \cdot k T \cdot C_{d}}{\omega_{f} \cdot t_{m}}
$$

where $k T$ is the Boltzmann energy, $C_{d}$ is the. detector capacitance, $\omega_{f}$ is the cut-off angular frequency of the first transistor in the preamplifier and $t_{m}$ is the peaking time of the weighting function. We will put $t_{d r i f t}=2 \cdot t_{m}$ to compare ENCs at the same speed of both detectors. For detectors of radii of $2 \mathrm{~cm}$ the ENCs of drift and classical detectors are 700 and 2000 electrons respectively. It is interesting to note that in order to improve the ENC of the drift detector we have to lower the value of $I_{\text {leak }}$, that is, to improve the technology of the detector itself. To improve the performance of the classical detector the $\omega_{f}$ has to be increased, that is, the speed of the external electronics has to be improved.

The negative side of memory detectors is that their electrode structure is more complex than the structure of classical detectors. Their production is more complicated and the cost is higher than the cost of classical detectors.

\section{REFERENCES}

1. K. McKay, Phys. Rev. $\underline{84}, 829$ (1951).

2. P. Holl et al., IEEE Trans. on Nuclear Science, 36, 251 (1989).

3. J. Walton and F. Goulding, IEEE Trans. on Nuclear Science, 34, 396 (1987).

4. W. Boyle and G. Smith, Bell System Technical Journal, $\underline{49}, 587$ (1970).

5. R. Bailey at al., Nucl. Instr. and Methods, 213, 201 (1983).

6. E. Gatti and P. Rehak, Nucl. Instr. and Meth., 225, 608 (1984).

7. L. Strüder et al., MPE Preprint 154, June 1989 to be published in Nucl. Instr. and Methods A in Proceedings of the Fifth European Symposium on Semiconductor Detectors, Munich, February 21-23.

8. J. Janesick et al., Astronomical Society of Pacific Conference Series No. 6, CCDs in Astronomy," ed. G. Jacoby, (Provo: Brigham Young University Press)1989. 
9... E. Gatti et al., Nucl. Instr. and Meth $, \frac{226}{\text {, }}, 129$ (1984).

10. P. Rehak et at., Nuct. Instr. and-Meth., A235, 224 (1985).-

11. P. Rehak at al., IEEE Trans. on Nuclear Science, 36,203 (1989).

12. V. Radeka et al., IEEE Trans. on Nuclear Science, $\underline{35}, 155$ (1988);

P. Rehak et al.,to be published in Nucl. Instr. and Methods A in Proceedings of the Fifth European Symposium on Semiconductor Detectors, Munich, February 21-23.

13. P. Rehak et al., Nucl. Instr. and Meth., $\underline{\text { A248, }} 367$ (1986).

\section{DISCLAIMER}

This icpurt was prepared as an account of work eponsored by an agency of the United States Government. Neither the United States Government nor any agency thereof, nor any of their employees, makes any warranty, express or implied, or assumes any legal liability or responsibility for the accuracy, completeness, or usefulness of any information, apparatus, product, or process disclosed, or represents that its use would not infringe privately owned rights. Reference herein to any specific commercial product, process, or service by trade name, trademark, manufacturer, or otherwise does not necessarily constitute or imply its endorsement, recommendation, or favoring by the United States Government or any agency thereof. The views and opinions of authors expressed herein do not necessarily state or reflect those of the United States Government or any agency thereof. 